\section{Fatores materno-infantis associados à diástase dos músculos retos do abdome no puerpério imediato}

\section{Factors relating to mother and child associated with separation of the rectus abdominis muscle in immediate puerperium}

\begin{abstract}
Objectives: to compare the distance between the rectus abdominis muscles (RAM) and the prevalence of separation of the rectus abdominis muscle (SRAM) in the supra- and infra-umbilical regions of primiparous and multiparous women during immediate post-partum and to identify associated factors relating to mother and child.

Methods: a cross-sectional study was carried out with 115 primiparous and 154 multiparous women. The SRAM was measured $4.5 \mathrm{~cm}$ above and below the umbilical scar and was correlated with maternal age, body mass index (BMI), gestational age, weight, height, and the circumference of the head and thorax of the newborn. Student's t test, Pearson's correlation, the chi-squared test and multiple logistic regression, $p<0.05$, were used to analyze the data.

Results: the distance between the supra-umbilical $R A M s$ was $3.04( \pm 1.68)$ and $3.35( \pm 1.19) \mathrm{cm}$ in the primiparous and multiparous women. The prevalence of supra-umbilical SRAM was $74.8 \%$ and $76.6 \%$ and that of infra-umbilical SRAM 40.0\% and $54.5 \%$. In both groups a positive correlation was found between the distance between the supra-umbilical RAM, the age of the mother, BMI and infra-umbilical RAM. The $B M I$ and weight of the newborn were the factors associated with supra-umbilical SRAM in primiparous women and maternal age was the associated factor among multiparous women.
\end{abstract}

Conclusions: irrespective of parity, the distance between the RAM and the prevalence of SRAM were greater in the supra-umbilical region and correlated with the age of the mother, BMI and infra-umbilical SRAM. Among primiparous women the associated factors were BMI and weight of the newborn and among multiparous women, the age of the mother alone.

Key words Postpartum period, Diastasis, Rectus abdominis, Parity, Infant, newborn
Mariana Tirolli Rett 1

Taíse Vasconcelos de Almeida 2

Andreza Carvalho Rabelo Mendonça 3

Josimari Melo DeSantana 4

Ana Paula de Lima Ferreira 5

Karina Conceição Gomes Machado de Araújo 6

1,2,5 Departamento de Fisioterapia. Hospital Universitário. Universidade Federal de Sergipe. Rua Claudio Batista s.n. Bairro Santo Antonio. Aracaju, SE, Brasil. CEP. 49.060-108.

E-mail: marianatrb@gmail.com

3,4 Programa de Pós-Graduação em Ciências da Saúde. Departamento de Medicina. Universidade Federal de Sergipe. Aracaju, SE, Brasil.

6 Departamento de Morfologia. Universidade Federal de Sergipe. São Cristóvão, SE, Brasil.

\section{Resumo}

Objetivos: comparar a distância entre os músculos retos do abdome (MRA) e a prevalência da diástase (DMRA) nas regiões supra-umbilical e infraumbilical de primíparas e multíparas no pós-parto imediato e verificar quais fatores materno-infantis podem estar associados.

Métodos: estudo transversal, incluindo 115 primiparas e 154 multiparas. Avaliou-se a DMRA 4,5 cm acima e abaixo da cicatriz umbilical. Correlacionouse com idade materna, indice de massa corporal $(I M C)$, idade gestacional, peso, estatura, perímetro cefálico e torácico do recém-nascido (RN). Utilizouse test $t$ de Student, correlação de Pearson, quiquadrado e regressão logística múltipla, $p<0,05$.

Resultados: a distância entre os MRA supraumbilical foi 3,04 $( \pm 1,68)$ e 3,35 $( \pm 1,19) \mathrm{cm}$ nas primiparas e multiparas. A prevalência da DMRA supra-umbilical foi $74,8 \%$ e $76,6 \%$ e, infra-umbilical $40,0 \%$ e $54,5 \%$. Nos dois grupos observou-se correlação positiva da distância entre os MRA supraumbilical com idade materna, IMC e infra-umbilical. $O I M C$ e peso do $R N$ foram fatores associados à DMRA supra-umbilical na primíparas e a idade materna nas multiparas.

Conclusões: independente da paridade, a distância entre os MRA e prevalência da DMRA foram maiores na região supra-umbilical e, correlacionouse com idade materna, IMC e infra-umbilical. Nas primiparas os fatores associados foram IMC e peso do RN e nas multiparas, a idade materna.

Palavras-chave Período pós-parto, Diástase, Reto do abdome, Paridade, Recém-nascido 


\section{Introdução}

O período gestacional é caracterizado por intensas adaptações físicas que podem repercutir no puerpério. Durante a gestação, o crescimento uterino, as mudanças hormonais e as adaptações biomecânicas favorecem o estiramento da musculatura abdominal. ${ }^{1-3}$ Isto, pode facilitar o afastamento entre os músculos retos do abdome (MRA) ou diástase dos músculos retos do abdome (DMRA), que é definida como o afastamento ou separação dos feixes desta musculatura ao longo da linha alba. ${ }^{1-3}$

Alguns fatores podem ser considerados predisponentes para o afastamento entre os MRA tais como gestações múltiplas, multiparidade, polihidrâmnio, macrossomia fetal e flacidez da musculatura abdominal pré-gravídica. 1,4-10 Mas, na literatura, existem divergências em relação à definição deste afastamento, tornando sua epidemiologia e fatores associados questionáveis. Alguns autores consideram qualquer afastamento entre os MRA,7 outros consideram como um afastamento superior a $2 \mathrm{~cm}^{10}$ ou $3 \mathrm{~cm} \cdot{ }^{1-3}$ Assim, dependendo do critério utilizado para avaliação, a DMRA pode ocorrer em $66,0 \%$ das mulheres durante a gestação $4,6,11,12$ e de 36,0 a $100,0 \%$ no pós-parto imediato.7,8,12-14 É mais facilmente percebida a partir do segundo trimestre da gestação, podendo ser uma situação transitória 5,15 ou permanente no pós-parto. 13,16

Clinicamente, a mensuração da DMRA pode ser feita manualmente por meio do número de polpas digitais entre as bordas mediais dos $\mathrm{MRA}^{8}$ ou utilizando paquímetro.3-6 A avaliação manual é um método simples, mas, devido à variação entre os dedos dos avaliadores, pode ser questionada, ${ }^{3}$ enquanto o paquímetro é mais objetivo, pois mensura a distância entre os MRA em milímetros ou centímetros. Quando é necessária a avaliação de alta precisão, exames de imagens como a ultrassonografia10 e a tomografia computadorizada17,18 também podem ser empregados. As medidas podem ser feitas nas regiões supra-umbilical e infra-umbilical,3,8,16 bem como na região umbilical.4,11-13,15,19

$\mathrm{O}$ afastamento entre os MRA e o desequilíbrio da musculatura abdominal podem levar à dor lombar, ao aparecimento de hérnia das vísceras abdominais $\mathrm{e}$ a disfunções uroginecológicas. 7,12,20 Com isso, tem sido questionado qual afastamento entre os MRA deve ser relevante, como deve ser avaliada esta distância e quais são suas repercussões, para que sejam desenvolvidas estratégias humanizadas do atendimento interdisciplinar em obstetrícia para prevenção e tratamento da DMRA, tanto no período gestacional quanto no pós-parto.
Embora sejam discutidos alguns fatores associados com o afastamento dos MRA, ,7,8,14,21 ainda não é claro se a paridade, idade gestacional e tamanho do feto, entre outras características podem repercutir na DMRA quando avaliada após o parto. Assim, os objetivos desse estudo foram comparar a distância entre os MRA e a prevalência da DMRA nas regiões supra-umbilical e infra-umbilical de primíparas e multíparas no pós-parto imediato, além deverificar quais fatores materno-infantis podem estar associados.

\section{Métodos}

Foi realizado um estudo observacional do tipo transversal, no período de agosto a novembro de 2011 na Maternidade Nossa Senhora de Lourdes (MNSL), localizada na cidade de Aracaju, Estado de Sergipe. No formulário do estudo utilizado para a coleta de dados foram registradas a partir do prontuário, informações maternas, como idade, índice de massa corporal (IMC), estado civil, profissão, doenças associadas à gestação e antecedentes obstétricos (paridade, tipo de parto, idade gestacional). Do recém-nascido (RN) foram registrados peso, estatura, perímetro cefálico e perímetro torácico.

As puérperas foram selecionadas mediante a verificação dos prontuários, respeitando um mínimo de 6 horas após o parto vaginal e 8 horas após a cesariana, conforme solicitação do local do estudo. Após a verificação do prontuário e dos critérios de inclusão e exclusão, as voluntárias foram esclarecidas sobre o Termo de Consentimento Livre e Esclarecidas (TCLE) e os objetivos da pesquisa. Foram incluídas puérperas primíparas e multíparas (no mínimo dois partos), submetidas a parto cesário ou vaginal. Foram excluídas puérperas com gestações múltiplas, índice de massa corporal $\geq 40$ $\mathrm{Kg} / \mathrm{m}^{2}$, idade gestacional $\leq 36$ semanas e prontuário incompleto. Em seguida, foi avaliada a distância entre os MRA, medida com a puérpera em decúbito dorsal, com o quadril fletido e joelhos flexionados a $90^{\circ}$, pés apoiados no leito e membros superiores estendidos paralelos ao corpo. Nessa posição, foi solicitada a flexão anterior do tronco em direção aos joelhos até que as espinhas das escápulas saíssem do leito. Dois pontos foram utilizados como referência para as medidas: $4,5 \mathrm{~cm}$ acima e 4,5 abaixo da cicatriz umbilical. Nesta posição, os dedos do avaliador foram posicionados de maneira perpendicular às bordas mediais dos MRA sendo o espaço entre os dedos imediatamente medido com paquímetro digital (Marca Western, escala de 0,01) com o registro final em centímetros. Foi considerada 
presença de DMRA um afastamento $\geq 2 \mathrm{~cm}$ na região supra-umbilical 13 e $\geq 1 \mathrm{~cm}$ na região infra-umbilical. 7,13

Os dados foram coletados por uma pesquisadora (TVA) devidamente treinada quanto à avaliação da distância entre os MRA. O estudo foi aprovado pelo Comitê de Ética em Pesquisa da Universidade Federal de Sergipe (UFS), ${ }^{\circ}$ CAAE 0015.0.107.000-11.

Para o cálculo amostral, foi utilizado o software EPIINFO 7.0. Considerando os critérios de inclusão/ exclusão, para um universo de 680 mulheres, prevalência estimada de $66,0 \%{ }^{7}$ de DMRA na região supra-umbilical e nível de confiança de $95 \%$, a amostra estimada foi de 229 mulheres. Para análise descritiva da amostra, foram realizadas medidas de tendência central (média), dispersão (desviopadrão), frequência e porcentagem. Foi utilizado o teste de Kolmogorov-Smirnov para verificar a normalidade das distribuições, dos dados, o teste $t$ de Student para comparar as variáveis numéricas; o qui-quadrado para comparação de proporções e o teste de correlação de Pearson para verificar a correlação das variáveis materno-infantis com a distância entre os MRA nos grupos de primíparas e multíparas. Adicionalmente foi realizada regressão logística múltipla para estimativa de risco pelo Odds Ratio (OR). Os dados foram analisados com o programa BioEstat 5.0, considerando-se o nível de significância de $p<0,05$ em todas as análises.

\section{Resultados}

Foram avaliadas 269 puérperas, sendo 115 (42,7\%) primíparas, das quais $59(51,3 \%)$ foram submetidas à cesariana e $56(48,7 \%)$ ao parto vaginal. Das 154 $(57,2 \%)$ multíparas, $69(44,8 \%)$ foram submetidas à cesariana e $85(55,1 \%)$ ao parto vaginal $(p=0,479)$.

Observou-se que a idade e o índice de massa corporal das multíparas foram significativamente superiores às mulheres primíparas $(p=0,001 \mathrm{e}$ $p=0,002$, respectivamente). A idade gestacional, peso, estatura, perímetro cefálico e perímetro torácico dos RNs não apresentaram diferença significativa entre os grupos (Tabela 1).

A distância média entre os MRA na região supraumbilical foi semelhante entre as primíparas 3,04 $( \pm 1,68) \mathrm{cm}$ e multíparas $3,35( \pm 1,19) \mathrm{cm}(p=0,13)$. $\mathrm{Na}$ região infra-umbilical, as multíparas apresentaram um afastamento dos MRA significativamente maior do que as primíparas $1,20( \pm 1,13) \mathrm{cm}$ e 0,82 $( \pm 0,74) \mathrm{cm}$ respectivamente $(p<0,01)$. Tanto nas primíparas quanto nas multíparas, a distância entre os MRA da região supra-umbilical foi significativamente maior do que na região infra-umbilical $(p<0,01)$ (Tabela 2).

Tabela 1

Características gerais e obstétricas das primíparas e multíparas.

\begin{tabular}{lccc}
\hline \multirow{2}{*}{ Características } & Primíparas (N=115) & Multíparas (N=154) & \multirow{2}{*}{$\boldsymbol{p}^{*}$} \\
\cline { 2 - 3 } & $\overline{\mathrm{X}} \pm \mathrm{DP}$ & $\overline{\mathrm{X}} \pm \mathrm{DP}$ & \\
\hline Características maternas & & & 0,01 \\
$\quad$ Idade materna (anos) & $22,05 \pm 5,80$ & $29,50 \pm 6,97$ & 0,02 \\
$\quad$ Índice de massa corporal (kg/m²) & $25,48 \pm 4,65$ & $27,93 \pm 5,22$ & \\
Características dos recém-nascidos & & & 0,75 \\
$\quad$ Idade gestacional (semanas) & $38,12 \pm 2,09$ & $38,20 \pm 2,03$ & 0,18 \\
Peso (g) & $2,591 \pm 0,447$ & $3,130 \pm 0,341$ & 0,28 \\
Estatura (cm) & $47,06 \pm 2,70$ & $46,82 \pm 3,34$ & 0,29 \\
Perímetro cefálico (cm) & $33,43 \pm 1,86$ & $33,82 \pm 1,99$ & 0,19 \\
$\quad$ Perímetro torácico $(\mathrm{cm})$ & $31,99 \pm 2,61$ & $32,56 \pm 2,64$ & \\
\hline * Teste t de Student. & & &
\end{tabular}

Tabela 2

\begin{tabular}{lcc}
\hline Comparação da distância entre os MRA supra-umbilical e infra-umbilical entre primíparas e multíparas. \\
\hline Distância entre os MRA (cm) & Primíparas (N=115) & Multíparas (N=154) \\
\cline { 2 - 3 } & $\bar{X} \pm$ DP & $\bar{X} \pm$ DP \\
\hline Supra-umbilical & $3,04 \pm 1,68$ & $3,35 \pm 1,19$ \\
Infra-umbilical & $0,82 \pm 0,74$ & $1,20 \pm 1,13$ \\
\hline
\end{tabular}

MRA= músculos retos do abdome; * Teste $t$ de Student. 
Segundo os critérios ( $\geq 2 \mathrm{~cm}$ na região supraumbilical $\mathrm{e} \geq 1 \mathrm{~cm}$ na infra-umbilical) foram descritas as prevalências de DMRA. Na região supra-umbilical, observou-se que a prevalência nas primíparas foi de $74,8 \%$ e nas multíparas de $76,6 \%$. Já na região infra-umbilical foi de $40,0 \%$ e $54,5 \%$, respectivamente. Não foi observada nenhuma diferença estatística entre as prevalências (Tabela 3 ).

Nas primíparas, a distância entre os MRA supraumbilical apresentou correlação positiva, porém fraca com a idade materna $(\mathrm{r}=0,24 ; p=0,007)$, o IMC $(\mathrm{r}=0,41 ; p<0,001)$, idade gestacional $(\mathrm{r}=0,28$; $p=0,02)$, o peso do $\mathrm{RN}(\mathrm{r}=0,36 ; p<0,01)$ e com a distância infra-umbilical $(\mathrm{r}=0,23 ; p=0,006)$. Nas multíparas, a distância entre os MRA supra-umbilical apresentou correlação positiva e fraca com a idade materna $(\mathrm{r}=0,27 ; p=0,006)$, o IMC $(\mathrm{r}=0,21$; $p=0,03)$ e a infra-umbilical $(\mathrm{r}=0,37 ; p=0,01)$ (Tabela $4)$.

Foram encontrados como fatores associados à DMRA supra-umbilical, o IMC $(p=0,04)$, o peso do $\mathrm{RN}(\mathrm{p}=0,04)$ e a DMRA infra-umbilical $(p=0,03)$ nas primíparas. Nas multíparas, apenas a idade materna $(p=0,03)$ e DMRA infra-umbilical $(p=0,01)$ estiveram associadas à DMRA supra-umbilical (Tabela 5).

Tabela 3

Prevalência de DMRA supra-umbilical e infra-umbilical nas primíparas e multíparas.

\begin{tabular}{|c|c|c|c|c|c|}
\hline \multirow{2}{*}{ DMRA } & \multicolumn{2}{|c|}{ Primíparas $(\mathrm{N}=115)$} & \multicolumn{2}{|c|}{ Multíparas ( $\mathrm{N}=154)$} & \multirow{2}{*}{$p^{*}$} \\
\hline & $\mathrm{n}$ & $\%$ & $\mathrm{n}$ & $\%$ & \\
\hline \multicolumn{6}{|c|}{ Supra-umbilical } \\
\hline$\geq 2 \mathrm{~cm}$ & 86 & 74,8 & 118 & 76,6 & 0,84 \\
\hline$<2 \mathrm{~cm}$ & 29 & 25,2 & 36 & 23,4 & \\
\hline \multicolumn{6}{|c|}{ Infra-umbilical } \\
\hline$\geq 1 \mathrm{~cm}$ & 46 & 40,0 & 84 & 54,5 & 0,73 \\
\hline$<1 \mathrm{~cm}$ & 69 & 60,0 & 70 & 45,4 & \\
\hline
\end{tabular}

* Teste qui-quadrado; DMRA= diástase dos músculos retos abdominais.

Tabela 4

Correlação linear da distância entre os MRA com as variáveis maternas e dos recém-nascidos.

\begin{tabular}{|c|c|c|c|c|}
\hline \multirow{2}{*}{ Distância entre os MRA supra-umbilicais } & \multicolumn{2}{|c|}{ Primíparas $(\mathrm{N}=115)$} & \multicolumn{2}{|c|}{ Multíparas $(\mathrm{N}=154)$} \\
\hline & $\mathrm{r}$ & $p$ & $\mathrm{r}$ & $p$ \\
\hline Idade materna (anos) & 0,24 & 0,007 & 0,27 & 0,006 \\
\hline Índice de massa corporal $\left(\mathrm{kg} / \mathrm{m}^{2}\right)$ & 0,41 & $<0,001$ & 0,21 & 0,03 \\
\hline Idade gestacional (semanas) & 0,28 & 0,02 & 0,11 & 0,15 \\
\hline Peso do recém-nascido (g) & 0,36 & $<0,01$ & 0,14 & 0,08 \\
\hline Estatura $(\mathrm{cm})$ & $-0,04$ & 0,66 & 0,05 & 0,52 \\
\hline Perímetro cefálico $(\mathrm{cm})$ & 0,00 & 0,94 & 0,04 & 0,59 \\
\hline Perímetro torácico $(\mathrm{cm})$ & $-0,18$ & 0,08 & 0,03 & 0,69 \\
\hline Distância MRA infra-umbilical (cm) & 0,23 & 0,006 & 0,37 & 0,01 \\
\hline
\end{tabular}

MRA= músculos retos do abdome; $r=$ coeficiente de correlação (Pearson). 
Fatores materno-infantis associados à DMRA supra-umbilical nas primíparas e multíparas.

\begin{tabular}{lcccc}
\hline \multirow{2}{*}{ DMRA supra-umbilical } & \multicolumn{2}{c}{ Primíparas } & \multicolumn{2}{c}{ Multíparas } \\
\cline { 2 - 5 } & OR (IC95\%) & $p$ & OR (IC95\%) & $p$ \\
\hline Idade materna (anos) & $0,98(0,90-1,07)$ & 0,70 & $1,07(1,01-1,15)$ & 0,03 \\
Índice de massa corporal (kg/m²) & $1,11(0,99-1,26)$ & 0,04 & $1,00(0,93-1,09)$ & 0,94 \\
Idade gestacional (semanas) & $1,05(0,84-1,32)$ & 0,64 & $1,20(0,95-1,53)$ & 0,11 \\
Peso do recém-nascido (g) & $2,89(0,95-4,76)$ & 0,04 & $0,99(0,47-2,11)$ & 0,98 \\
Estatura (cm) & $0,99(0,80-1,23)$ & 0,95 & $1,00(0,88-1,14)$ & 0,98 \\
Perímetro cefálico (cm) & $0,93(0,70-1,26)$ & 0,68 & $0,90(0,74-1,12)$ & 0,38 \\
Perímetro torácico (cm) & $0,93(0,77-1,14)$ & 0,51 & $0,92(0,78-1,10)$ & 0,37 \\
DMRA infra-umbilical & $7,98(2,61-24,39)$ & 0,03 & $2,30(1,38-3,86)$ & 0,01 \\
& & & & \\
\hline
\end{tabular}

DMRA= diástase dos músculos retos abdominais; Análise: regressão logística múltipla; OR=odds ratio.

\section{Discussão}

A prevalência da distância entre os MRA pode variar de $35,0 \%$ a $100,0 \%, 7,12-14$ dependendo dos critérios e do local de avaliação. No presente estudo, a ocorrência da DMRA nas primíparas e multíparas foi de $74,9 \%$ e $76,6 \%$ na região supra-umbilical e de $40,0 \%$ e $54,5 \%$ na infra-umbilical, respectivamente. Tais valores estão próximos ao relatados por autores que adotaram critérios semelhantes, incluindo uma maior frequência na região supra-umbilical.8,9,12,14

Segundo Berr et al.,21 e Coldron et al.,22 em nulíparas avaliadas com ultrassonografia a média da distância entre os MRA supra-umbilical foi de 1,3 $\mathrm{cm}$ e $1,1 \mathrm{~cm}$, respectivamente. É possível que a média da distância entre os MRA do presente estudo foi superior $(3,04$ e $3,35 \mathrm{~cm})$, por se tratar de primíparas e multíparas. Contudo, independentemente da paridade, a média da distância e a prevalência da DMRA foram maiores na região supra-umbilical quando comparada com a infra-umbilical, dados consoantes com a literatura. $3,4,8,15$

Na região infra-umbilical a distância entre os MRA pode ser menor pela diferença anatômica existente entre as disposições das fáscias que recobrem o músculo retoabdominal porque acima da cicatriz umbilical, há um folheto anterior (formado pela aponeurose de inserção do oblíquo externo e pela delaminação anterior da aponeurose do oblíquo interno) e um posterior (formado pela delaminação posterior da aponeurose do oblíquo interno e pela aponeurose de inserção do transverso). Abaixo, só existem folhetos anteriores que são formados por três músculos. 23 Assim, a porção final desse músculo é mais forte, dificultando o afastamento dos seus ventres.

Assim como achados anteriores, 8,24 a distância entre os MRA supra-umbilical foi similar entre os grupos, embora nas multíparas tenha sido discretamente superior. Tal fato pode ser devido ao efeito apenas da última gestação ou até da fraqueza abdominal pré-gestacional existente nos dois grupos. Entretanto, a distância entre os MRA na região infraumbilical foi significativamente maior nas multíparas, o que também já foi encontrado anteriormente. $3,8,13 \mathrm{O}$ maior afastamento destes músculos em multíparas pode ser devido ao efeito acumulativo das gestações, o peso e as dimensões do útero, que em constante aumento, influenciam a morfologia musculoesquelética do tronco, aumentando as distâncias entre as inserções musculares.

Chama a atenção o fato de ter sido observado que o IMC e o peso do RN foram considerados fatores associados entre as primíparas e somente a idade materna para as multíparas, ao mesmo tempo em que as multíparas apresentaram idade e IMC significativamente superiores em relação às primíparas. Talvez, o ganho de peso na primeira gestação seja um fator que tenha um impacto maior do que o ganho de peso em outras gestações, pela adaptação corporal inicial da primigesta.

Contudo, observou-se correlação positiva, embora fraca, da idade e IMC com a distância entre os MRA supra-umbilical nas primíparas e multíparas. Isso nos leva a refletir que o afastamento destes músculos não está somente relacionado à paridade, mas ao aumento da idade e do IMC. A idade materna foi o único fator associado entre as multíparas. Mulheres com mais idade, provavelmente têm antecedentes de maior número de gestações e partos, 
o que potencializa e facilita o aparecimento da DMRA, tanto na região supra quanto infraumbilical.7,13,12,14,24-26 Em estudos anteriores, mulheres com idade mais avançada e maior número de gestações e de partos, apresentaram uma maior prevalência de distância entre os MRA. ${ }^{7} \mathrm{O}$ estiramento sucessivo da musculatura abdominal promovido pelas gestações, juntamente com a idade materna, podem promover maior flacidez e abertura dos retos do abdome. Ainda entre as multíparas, embora não tenham sido fatores associados, o peso do RN e o IMC elevado podem potencializar tal situação, talvez por maior distensão uterina. Souza et al. 14 encontraram que a multiparidade pode influenciar em $64,0 \%$, a faixa etária materna em $43,0 \%$ e peso do RN em 11,0\% na DMRA. Mas, não se deve descartar o fato de o IMC e o peso do RN estarem associados no grupo das primíparas.

A maior parte da amostra apresentou uma distância dos MRA na região supra-umbilical superior a $2,0 \mathrm{~cm}$, conforme verificado em estudos anteriores que também envolveram puérperas. $8,9,14,16$ Um afastamento acima de 2,5 a $3,0 \mathrm{~cm}$ pode ser considerado prejudicial, $3,7,15$ pois pode interferir nas funções da musculatura abdominal como estabilização do tronco, postura, respiração, defecação, trabalho de parto e apoio das vísceras abdominais. $5,13 \mathrm{~A}$ atividade eletromiográfica da musculatura abdominal demostrou que a DMRA pode ter influência na geração de esforços voluntários durante o período expulsivo do parto. $27 \mathrm{Um}$ déficit na estabilização do tronco pode predispor ao desenvolvimento de lombalgia, hérnias abdominais e disfunções uroginecológicas como a incontinência urinária, incontinência fecal e prolapso dos órgãos pélvicos.7 $\mathrm{E}$, mulheres submetidas à abdominoplastia para redução da DMRA podem obter alívio significativo da dor lombar, evidenciando a relação entre estas musculaturas. 8

O fato de mais de $70,0 \%$ das puérperas terem apresentado um afastamento $\geq 2 \mathrm{~cm}$ e da média desta distância na região supra-umbilical estar acima de $3,0 \mathrm{~cm}$, pode indicar que são candidatas às disfunções provenientes da falta de estabilização abdominal e merecem atenção especial com relação a realização de exercícios abdominais no pós-parto. Acredita-se que haja uma redução parcial deste afastamento até a $8^{\mathrm{a}}$ semana pós-parto, mas nem todas as situações são resolvidas espontaneamente e nem sempre retornam aos valores pré-gravídicos. ${ }^{22}$ Isto justifica e reforça a necessidade de ser realizada a avaliação criteriosa deste afastamento entre os
MRA, para o planejamento adequado da conduta terapêutica. A realização de exercícios físicos devidamente supervisionados, tanto no período gestacional quanto no pós-parto, são importantes não só para tratar disfunções musculoesqueléticas, mas também para prevenção. O fortalecimento dos músculos abdominais durante o pré-natal e no puerpério imediato parece ter efeito positivo na diminuição da DMRA. ${ }^{3,13}$

Embora tenha sido observado que apenas a idade materna foi fator associado entre a multiparidade e $o$ IMC e o peso do RN, nas primíparas, encontrou-se correlação positiva da distância entre os MRA supraumbilical com a idade materna, IMC e DMRA infraumbilical em ambos os grupos. Apesar de terem sido verificadas correlações significativas, a força da correlação foi fraca, o que pode indicar que outros fatores não avaliados possam também interferir. Mesmo assim, há uma responsabilidade dos profissionais da saúde que se preocupam com a funcionalidade e a integridade física das puérperas, em orientar sobre as chances, os fatores associados e os riscos de DMRA em uma gestação posterior, sobre o ganho de peso materno e fetal durante a gestação. Por exemplo, mulheres com diabetes gestacional que tem maior probabilidade de feto macrossômico e gestações múltiplas, devem ser esclarecidas.

Avaliar adequadamente a musculatura abdominal é importante para orientar o cuidado interdisciplinar e humanizado em obstetrícia, além de facilitar a comunicação entre pesquisadores e profissionais da área da saúde. Na presença da DMRA devem ser recomendados exercícios específicos na tentativa de evitar problemas futuros, como, por exemplo, piora da DMRA em uma próxima gestação ou comprometimento de funções uroginecológicas. 7

O presente estudo foi conduzido com mulheres no pós-parto imediato e isto limita inferências sobre $\mathrm{o}$ assunto no período gestacional, puerpério remoto e tardio. Além disso, considerou o IMC após o parto e não o ganho de peso ponderal na gestação, que pode também influenciar os resultados. Isso se deve à falta desta informação pelas poucas consultas pré-natal e cartões incompletos dessas mulheres que no Brasil, são todas atendidas pelo Sistema Único de Saúde. ${ }^{29}$ Como são escassos os estudos que correlacionem a DMRA com variáveis materno-infantis, espera-se que os resultados possam contribuir para a elaboração de estratégias de prevenção e tratamento da DMRA, e para o atendimento em centros especializados na saúde materna e infantil. 


\section{Agradecimentos}

Às puérperas, à Maternidade Nossa Senhora de Lourdes e Maternidade Santa Isabel. À Fundação de Apoio à Pesquisa e à Inovação Tecnológica do

\section{Referências}

1. Machado AV. Puerpério. In: Baracho E, editor. Fisioterapia aplicada à obstetrícia, uroginecologia e aspectos de mastologia. Rio de Janeiro: Guanabara Koogan; 2007. p. 225-40.

2. Artal R, O'Toole M, White S. Guidelines of the American College of Obstetricians and Gynecologists for exercise during pregnancy and the postpartum period. Br J Sports Med. 2003; 37 (1): 6-12.

3. Vasconcelos MS, Lemos A. Distância dos músculos retos do abdome no ciclo gravídico puerperal. In: Lemos A, editor. Fisioterapia obstétrica baseada em evidências. Rio de Janeiro: MedBook; 2013. p.187-98.

4. Lemos A, Souza AI, Andrade AD, Figueiroa JN, CabralFilho JE. Pregnancy inter-recti abdominis distance has no impact on respiratory strength. J PhysTher Sci. 2011; 23 (5): 757-60.

5. Mesquita LA, Machado AV, Andrade AV. Fisioterapia para a redução da diástase dos músculos reto abdominais no pósparto. Rev Bras Ginecol Obstet. 1999; 21(5): 267-72.

6. Lemos A, Caminha, MA, Melo Jr., EF, Andrade DA Avaliação da força muscular respiratória no terceiro trimestre de gestação. Rev Bras Fisioter. 2005; 9 (2): 15156.

7. Spitznagle TM, Leong FC, Van Dillen LR. Prevalence of diastasis recti abdominis in aurogynecological patient population. Int Urogynecol J Pelvic Floor Dysfunct. 2007; 18 (3): 321-8.

8. Rett MT, Braga MD, Bernardes NO, Andrade SC. Prevalence of diastasis of the rectus abdominis muscles immediately postpartum: comparison between primiparae and multiparae. BrazJ PhysTher. 2009; 13 (4): 275-80.

9. Bursch SG. Interrater reliability of diastasis recti abdominis measurement. PhysTher. 1987; 67 (7): 1077-79.

10. Mendes DA, Nahas FX, Veiga DF, Mendes FV, Figueiras RG, Gomes HC, Ely PB, Novo NF, Ferreira LM. Ultrasonography for measuring rectus abdominis muscles diastasis.Acta Cir Bras. 2007; 22 (3): 182-6.

11. Hsia M, Jones S. Natural resolution of rectus abdominis diastasis. Two single case studies. Aust J Physiother. 2000; 46 (4): 301-7.

12. Boissonnault JS, Blaschak MJ. Incidence of diastasis recti abdominis during the childbearing year. PhysTher. 1988; 68: 1082-6.

13. Chiarello CM, Falzone LA, McCaslin KE, Patel MN, Ulery KR. The effects of as exercise program on diastasis recti abdominis in pregnant women. J Women's Health Phys Ther. 2005; 29 (1): 11-6.
Estado de Sergipe (FAPITEC/SE) e ao Programa Institucional de Bolsas de Iniciação à Extensão (PIBIX/PROEX//UFS) pelo incentivo e apoio financeiro.

14. Souza CAAR, Oliveira RA, Lima ACG. Diástase dos músculos reto abdominais em puérperas na fase hospitalar. Fisioter Bras. 2009; 10 (5): 333-8.

15. Gilleard WL, Brown JM. Structure and function of the abdominal muscles in primigravid subjects during pregnancy and the immediate postbirth period. Phys Ther. 1996; 76 (7): 750-62.

16. Boxer $\mathrm{S}$, Jones $\mathrm{S}$. Intra-rater reliability of rectus abdominis diastasis measurement using dial calipers. Aust J Physiother. 1997; 43 (2): 109-14.

17. Rath AM, Attali P, Dumas JL, Goldlust D, Zhang J, Chevrel JP. The abdominal linea alba: an anatomoradiologic and biomechanical study. Surg Radiol Anat. 1996; 18 (4): 281-

18. Lockwood D, Einstein D, Davros W. Diagnostic imaging: radiation dose and patients's concerns. Cleve Clin J Med. 2006; 73 (6): 583-6.

19. Huge BS, Kisner C. Princípios de exercícios para a paciente obstétrica. In: Kisner, C, Colby LA. Exercícios Terapêuticos: Fundamentos e Técnicas. 5 ed. São Paulo: Manole; 2009; p. 843-44.

20. Toranto IR. The relief of low back pain with the WARP abdominoplasty: a preliminary report. Plas Reconstr Surg. 1990; 85 (4): 545-55.

21. Beer GM, Schuster A, Seifert B, Manestar M, Mihic-Probst $\mathrm{D}$, Weber SA. The normal width of the lineaalba in nullíparous women. Clin Anat. 2009; 22: 706-11.

22. Coldron Y, Stokes MJ, Newham DJ, Cook K. Postpartum characteristics of rectus abdominis on ultrasound imaging. Man Ther. 2008; 13: 112-21.

23. Moore KL. O abdome. In: Moore KL, editor. Anatomia orientada para a clínica. 3 ed. Rio de Janeiro: Guanabara Koogan; 1994. p. 117-23.

24. Rett MT, Araújo FR, Rocha I, Silva RA. Diástase dos músculos retoabdominais no puerpério imediato de primíparas e multíparas após o parto vaginal. Fisioter Pesq. 2012; 19 (3): 236-41.

25. Lo T, Candido G, Janssen P. Diastasis of the recti abdominis in pregnancy: risk factors and treatment. Phys Can. 1999; 32-7.

26. Turan V, Colluoglu C, Turkyilmaz E, Korucuoglu U. Prevalence of diastasis recti abdominis in the population of young multiparous adults in Turkey. Ginekol Pol. 2011; 82 (11): 817-21. 
27. Oliveira BD, de Andrade AD, Lemos A, Brito VC, Pedrosa ML, Silva TN. Abdominal muscle electrical activity during labor expulsive stage: a cross-sectional study. Rev Bras Fisioter. 2011; 15 (6): 445-51

Recebido em 17 de maio 2013

Versão final apresentada em 12 de novembro de 2013

Aprovado em 11 de dezembro de 2013
28. Gurgel, RQ, Nery AMDG, Almeida MLD, Oliveira ERR, Lima DDF, Bettiol H, Barbieri MA. Características das gestações, partos e recém-nascidos da região metropolitana de Aracaju, Sergipe, Brasil. Rev Bras Saúde Matern Infant. 2009; 9 (2): 167-177. 